\section{The wrong drug}

Patients are sometimes given either the wrong drug or an excessive amount of the right one. Anaesthetists are at especial risk of such errors, since not only do they prescribe drugs but they also administer them; many of their drugs are potent; and the polypharmacy of modern anaesthesia increases the possibilities of drug interactions. Should an emergency arise during anaesthesia (or resuscitation, when anaesthetists are often present) there may not be the normal check on what drugs the patient is having. Finally, some drugs used by anaesthetists may be prepared for them by non-nursing ancillary staff. In these circumstances errors can be dangerous and they sometimes cost patients their lives. The Medico-Pharmaceutical Forum has just published ${ }^{1}$ some recommendations intended to reduce these risks, and anaesthetists should look at their routine practice in the light of these warnings.

How do mistakes occur? Verbal prescriptions are notorious as sources of error. Doctors' writing, too, is often illegible; and even prescription sheets on the London Hospital pattern do not remove all hazards, especially with the "once only" or "as needed" sections.

More serious are the errors in administration. Nowadays drugs reach patients through a complex process. A bulk supply to the pharmacy is broken into smaller packs and sent to the theatre. The ampoules may then be used individually, transferred to a second pack on an anaesthetic machine, or-worst of all-taken out, not used, and returned to one of the packs. Each handling carries a risk of error and the more the handling the more the probabilities multiply. Finding adrenaline ampoules in atropine packs is not unknown and may cause deaths.

Next is the problem of different drugs with similar names, a problem that may be compounded by using proprietary names. When a pharmacy changes its supplier there may be further complications-suxamethonium may change from Scoline to Anectine, producing confusion-and possibly leading to its use instead of atropine. Similarly, changes of neostigmine from amber-coloured to clear ampoules has caused confusion and irritation if nothing worse.

What can be done to minimise the risks? Only the most extreme emergency should allow verbal prescriptions to be accepted. The hazard of prescribing a neuromuscular blocking drug such as pancuronium to "settle" patients on ventilators without cancelling the prescription once the patient is breathing spontaneously derives from the anaesthetist alone. If such a patient is given pancuronium subsequently he has only himself to blame.

The problems of incorrect administration can be minimised by thought and some action from the pharmaceutical industry. The labelling of ampoules could be improved, and the MedicoPharmaceutical Forum suggests a format with the approved name, the proprietary name, and the amount of drug in the volume of content of the ampoule (for example, "Gallamine, B P, Flaxedil, $80 \mathrm{mg}$ in $2 \mathrm{ml}$ "). Concentrations should not be given, and paper labels should be replaced by ceramic labels as far as possible. Pharmacies and anaesthetists should decide how to keep drug transfers to a minimum. Despite the cost the practice of returning ampoules to pharmaceutical packs must be forbidden. Finally, the person administering the drug (the anaesthetist) should have the responsibility of drawing up the drug from the ampoule-the label of which he has read. Delegation to nursing or operating department staff should not be allowed, except in emergencies such as cardiac arrest. This may mean that the anaesthetist spends more time in theatre, but errors would be reduced. In 1978 the Medical Defence Union ${ }^{2}$ described six errors of injection; two were related to anaesthesia, and both led to the death of the patients.

\footnotetext{
1 Medico-Pharmaceutical Forum, Dangerous Mistakes in Drug Administration. London, Royal Society of Medicine, 1979.
}

${ }^{2}$ Medical Defence Union, Annual Report 1978. London, MDU, 1978.

\section{Acute renal failure; hyperuricaemia, and myoglobinuria .}

Patients with severe renal failure, whatever the cause, may have a raised serum uric acid concentration and in chronic renal failure this may result occasionally in secondary gout. In an increasing number of illnesses, however, there are reports of serum uric acid concentrations that are out of proportion to the degree of renal failure and are thought to be its cause. They fall into two broad categories, characterised on the one hand by massive destruction of leukaemic or lymphoma cells and on the other by damage or necrosis of skeletal muscle-in both of which uric acid is a metabolic breakdown product, though its role in the second group is more controversial.

Hyperuricaemic acute renal failure is a well-recognised occurrence with leukaemias and lymphomas-either as a rare spontaneous complication or, more usually, as a complication of intensive chemotherapy. The pathogenesis, however, has changed over the years. ${ }^{1}$ The first cases reported ${ }^{2}$ were due to obstruction of the ureters by uric acid crystals, and in these patients a brisk diuresis followed retrograde ureteric catheterisation and lavage. At this time the acute leukaemias and lymphomas had a high death rate and chemotherapy was in its infancy. Thus only those with more chronic disease survived long enough to develop ureteric obstruction due to prolonged hyperuricaemia and hyperuricosuria. In a review going up to $1966^{3}$ about one-third of the patients had symptoms suggestive of urinary tract obstruction. But by the time Kjellstrand et $a l^{4}$ reviewed the condition in 1974 the picture had changed. Chemotherapy for the leukaemias and lymphomas had improved considerably, and the more aggressive chemotherapy resulted in massive cell destruction, with release of large amounts of nucleic acid and dramatic rises in serum uric acid concentrations. Thus in the 16 patients they reviewed the mean plasma uric acid concentration was $1.19 \mathrm{mmol} / 1 \quad(20 \mathrm{mg} / 100 \mathrm{ml})$-range $0.71-2.14 \mathrm{mmol} / 1$ (12$36 \mathrm{mg} / 100 \mathrm{ml}$ )-at the time of acute renal failure, which was considered to be due to precipitation of uric acid crystals in the distal tubules and collecting ducts. In only one of the 16 cases was there any suggestion of obstruction of the ureter.

Besides the changing pathogenesis there has also been a notable improvement in the management and treatment of acute renal failure, with a greatly improved prognosis. Fourteen of 30 patients reported by $\mathrm{Lilje}^{3}$ died during the acute renal failure, but all 16 patients reviewed by Kjellstrand et al survived it. Kjellstrand $e t$ al have summarised the principles of management. Clearly the doctor should anticipate acute hyperuricaemia during chemotherapy in patients with leukaemias and lymphomas, and start treatment with allopurinol well before chemotherapy to minimise the rise in uric acid concentration. Patients must not become dehydrated, and alkalinisation of the urine during intense chemotherapy 
is advisable to reduce the proportion of insoluble uric acid in the urine. The dosage of allopurinol may need to be increased at such times. The risk of xanthine nephropathy from allopurinol treatment is remote. ${ }^{5}$ Hyperphosphataemiaagain presumably due to massive cell necrosis-is also important in some of these patients, ${ }^{4}$ and they may need oral aluminium hydroxide gel during intensive chemotherapy to minimise the possibility of metastatic calcification.

Urgent rehydration and alkalinisation of the urine are the first lines of treatment for hyperuricaemic acute renal failure, provided the diagnosis is made at an early stage and before acute renal failure and oliguria are established. Once oliguric acute renal failure is established, however, the most effective treatment is haemodialysis. This was shown by Kjellstrand et al to be 10-20 times more effective than peritoneal dialysis at removing uric acid and lowering the serum uric acid concentration. The patients they reviewed all survived the acute renal failure and recovered good renal function in three weeks or less. The possibility of bilateral ureteric obstruction should always be borne in mind, and excluded if there is anything to suggest it. Cystoscopy, retrograde ureteric catheterisation, and retrograde pyelography should not often be necessary these days, because in many cases urinary tract obstruction can be excluded by high-dose intravenous urography or gamma-camera renography, or both.

Several other clinical conditions and circumstances may occasionally be associated with acute renal failure, evidence of skeletal muscle injury or necrosis being the common factor. They include unaccustomed strenuous muscular exertion or heat exhaustion, or both ${ }^{6}$; repeated seizures; prolonged coma (caused by, for example, carbon monoxide, barbiturates, or alcohol); trauma (crush injuries); burns; electric shock; arterial occlusion; sea-snake bites; polymyositis (rarely); Meyer-Betz paroxysmal myoglobinuria; McArdle's syndrome; and Haff's disease. There are probably several causes for the acute renal failure in these circumstances, postulated ones including hypotension, dehydration, thermal damage, hypokalaemia, rhabdomyolysis and myoglobinuria, and hyperuricaemia. ${ }^{78}$

Myoglobinuria associated with the acute renal failure has been well documented in many of these conditions. The mechanism of myoglobin nephrotoxicity, however, is not clear. Myoglobinuria alone does not usually lead to renal failure and it can be detected in a high proportion of athletes after severe exertion, ${ }^{910}$ though the degree of muscle damage and myoglobinuria is more severe in acute renal failure associated with unaccustomed strenuous exercise. ${ }^{11} 12$ In animals, moreover, acute renal failure due to myoglobin (or haemoglobin) is more likely when other factors, such as hypotension and fluid depletion, are present. ${ }^{13} 14$

The acute renal failure that may accompany rhabdomyolysis, whether traumatic or non-traumatic, is associated with the usual biochemical features, but excessive breakdown of skeletal muscle results in serum potassium, phosphate, and uric acid concentrations that are higher and a serum calcium concentration that is lower than is usual in renal failure. In the diuretic phase transient hypercalcaemia may occur. The hypocalcaemia seems to be due to deposition of calcium within the damaged muscles, ${ }^{15}$ and the hypercalcaemia during the diuretic phase to mobilisation of the calcium from the muscles. Other features include clinical and biochemical evidence of muscle damage, with raised serum transaminase, creatinine phosphokinase, and lactic dehydrogenase concentrations. Myoglobinuria should be suspected when the urine is dark and positive to orthotolidine but contains no red blood cells. It has not always been found in these types of acute renal failure; but myoglobin is rapidly excreted and must be looked for during the first $24-48$ hours. It is reliably identified by acrylamide gel electrophoresis or immunodiffusion techniques. Spectrophotometry is less reliable, particularly in an acid urine. ${ }^{8}$

The role of hyperuricaemia in causing the acute renal failure in these circumstances is not clear, but recent reports suggest that it may be important in those cases that occasionally follow unaccustomed strenuous muscular exercise or heat exhaustion and repeated grand mal fits. ${ }^{16-19}$ Evidence of non-traumatic rhabdomyolysis and myoglobinuria after repeated fits was found in four patients reported by Grossman et $a l,{ }^{8}$ but they did not study serum uric acid concentrations. Warren et al ${ }^{18}$ considered that severe hyperuricaemia was chiefly responsible for the acute renal failure in their cases that followed epileptic fits, and they found no evidence of myoglobinuria (as judged by Haematest results). Lühdorf et $a l^{19}$ documented significant increases in serum uric acid concentrations after repeated grand mal fits and observed renal impairment in two cases, but they did not report on the presence or absence of myoglobin in the urine. Two mechanisms probably underlie the hyperuricaemia that may follow strenuous exercise or heat exhaustion and grand mal fits: increased production of uric acid as a result of skeletal muscle damage and necrosis, and reduced renal excretion of uric acid because of the lactic acidosis ${ }^{20}$ that may follow strenuous exercise or grand mal fits. ${ }^{16} 21$ The metabolic acidosis will also predispose to uric acid crystalluria caused by the acid urine.

Clearly we must be aware of the possibility-however rareof severe hyperuricaemia and acute renal failure after repeated grand mal fits. The serum uric acid concentration should be monitored closely and dehydration avoided or corrected. Alkalinisation of the urine should be considered if the serum uric acid concentration rises. Patients with established acute renal failure, however, appear to have a good prognosis with haemodialysis.

1 Lancet, 1974, 1, 1266.

2 Bedrna, J, and Polcak, J, Medizinische Klinik, 1929, 25, 1700.

${ }^{3}$ Lilje, E, Ugeskrift for Laeger, 1970, 132, 12.

${ }_{4}^{4}$ Kjellstrand, C M, et al, Archives of Internal Medicine, 1974, 133, 349.

5 Band, P R, et al, New England fournal of Medicine, 1970, 283, 354.

${ }^{6}$ Pusey, C D, Flynn, C T, and Winfield, C R, Fournal of the Royal Army Medical Corps, 1977, 134, 88.

7 Lancet, 1976, 2, 1343.

8 Grossman, R A, et al, New England fournal of Medicine, 1974, 291, 807.

${ }^{9}$ Ono, I, Tohoku fournal of Experimental Medicine, 1953, 57, 273.

${ }^{10}$ Eliot, R S, Shafer, R B, and Gibas, M A, Archives of Physical Medicine and Rehabilitation, 1967, 48, 229.

11 Jackson, R C, Proceedings of the Royal Society of Medicine, 1970, 63, 566.

12 Raju, S F, Robinson, G H, and Bower, J D, Southern Medical fournal, $1973,66,330$.

13 Thiel, G, McDonald, F D, and Oken, D E, Nephron, 1970, 7, 67.

14 Maluf, N S R, Annals of Surgery, 1949, 130, 49.

15 Akmal, M, et al, Kidney International, 1978, 14, 644.

16 Schrier, R W, et al, Annals of Internal Medicine, 1970, 73, 213.

17 Knochel, J P, Dotin, L N, and Hamburger, R J, Annals of Internal Medicine, 1974, 81, 321.

18 Warren, D J, Leitch, A G, and Leggett, R J E, Lancet, 1975, 2, 385.

19 Lühdorf, K, Petersson, H, and Pedersen, K, Acta Neurologica Scandinavica, 1978, 58, 280.

20 Yü, T F, et al, Proceedings of the Society for Experimental Biology and Medicine, 1957, 96, 809.

21 Orringer, C E, et al, New England fournal of Medicine, 1977, 297, 796. 